Part of Journal of Research of the National Bureau of Standards, Volume 28, February 1942

\title{
FURTHER INVESTIGATIONS OF THE AFFINITIES OF ANIONS OF STRONG ACIDS FOR WOOL PROTEIN
}

\author{
By Jacinto Steinhardt, ${ }^{1}$ Charles H. Fugitt, ${ }^{1}$ and Milton Harris ${ }^{1}$
}

\section{ABSTRACT}

Titration curves of wool with 18 strong acids at $0^{\circ}, 25^{\circ}$, or $50^{\circ} \mathrm{C}$ have been added to the data for 19 others presented earlier. Several have been investigated at more than one temperature. The reversibility of the equilibria measured has been demonstrated quantitatively. New anion-wool dissociation constants, based on modifications of equations previously used to calculate anion-wool affinities, are tabulated for 33 anions, and heats of dissociation of a few anionwool complexes are also given. The previously reported tendency of affinity to rise with molecular-weight is confirmed; fairly consistent relationships between the affinity and the molecular weights of strong organic acids appear.

\section{CONTENTS}

I. Introduction

II. Experimental procedure

1. Wool

2. Source of acids and specific procedures.

3. Measurement of reversibility of the combination with acids - -

4. Allow ance for the effects of hydrolytic decomposition

III. Results and discussion

1. Titration curves

2. The scale of anion affinities

3 . Effect of temperature on affinity.

202

203

205

IV. Relation of chemical structure to affinity

V. References

\section{INTRODUCTION}

It has been shown in a previous paper that the titration curves of wool and of other proteins obtained with a number of strong acids are not identical but occupy very widely different positions with respect to the $\mathrm{pH}$ coordinate [16]. ${ }^{2}$ These wide differences have been interpreted as due to combination of protein with anions as well as with hydrogen ions, the combination with each anion being governed by its own distinct dissociation constant. It has been shown also that by making a number of simple assumptions these dissociation constants (or their reciprocals, the affinities) may be calculated from the titration data.

The existence of differences in the affinities of anions for protein has a direct bearing on dyeing theory. A serious difficulty in "chemical" theories of dyeing, which have attempted to draw a close analogy between the combination of fibers with hydrochloric acid and their combination with acid dyes, has been the lack of an explanation of

\footnotetext{
${ }_{1}^{1}$ Research Associates at the National Bureau of Standards, representing the Textile Foundation.

2 Figures in brackets indicate the literature references at the end of this paper.
} 
why the combination of dyes is relatively fast to water and dilute alkalies while that of hydrochloric acid is not. If the equilibrium of fibers with both of these acids is considered solely in terms of an acidbase (i. e., hydrogen ion) equilibrium, one would conclude that both acids should be bound equally well. The existence of a second dissociation equilibrium involving each anion provides a basis for understanding these differences, and encourages exploitation of the many attractive features of the "chemical" theory of acid dyeing.

Experiments reported elsewhere [14] have shown that the quantitative formulation of the anion affinity concept may be applied to mixtures of acids or of acids and salts, systems which more nearly approach in complexity the dyebaths commonly employed in industry than do the simple systems involved in measurement of titration curves. Measurements of the amounts of each of the anions combined with the fiber have indicated the nature of some of the factors controlling fastness and degree of exhaust. In addition, they have suggested a consistent explanation of the part played by various widely used assistants (such as Glauber's salt) in promoting level or solid dyeing, which will be discussed elsewhere. Since it is indicated by these experiments that the relative effectiveness of such assistants is directly related to their affinities, it has appeared particularly important to determine the affinities of a still larger number of compounds of more diverse types than those previously investigated in order to establish with greater certainty the relation between affinity, size, and structure of ions. Such information, especially if it is combined with more detailed knowledge of the variation of affinity at higher temperatures, would provide a basis for selecting economically practical leveling agents having the desired range of affinities for wool.

The present paper, besides describing measurements of the affinity for wool of the anions of an additional number of strong acids, makes use of certain refinements in the methods of calculating affinity [11] which make possible a more detailed comparision of theoretical predictions with experimental data. On the basis of these refinements, the data previously presented are recomputed and a recapitulation is made of the results obtained with 33 strong acids. As a result, the discussion of the relation between chemical structure and affinity in this paper is based on a broader survey than was previously available.

\section{EXPERIMENTAL PROCEDURE}

Methods of purification of the wool, measurement of $\mathrm{pH}$ values, and determination of the amounts of acid combined have been described in earlier publications $[12,15,16]$. Additional details concerning the specific materials and procedures used in the present investigation are described in the following subsections.

\section{WOOL}

All of the new measurements described in this paper were made with a single lot of purified wool having a slightly higher acid-binding capacity than the wool used in earlier work. In order to allow for this small difference (about 0.03 millimole), the $\mathrm{pH}$ at which 0.415 millimole per gram is combined has been taken as the "midpoint $\mathrm{pH}$," by means of which the position of each curve with respect to the $\mathrm{pH}$ coordinate has been expressed, in place of the $\mathrm{pH}$ at which 0.40 
millimole is combined, which was previously used. When this is done, the half-combination points of curves obtained on both lots of wool with the same acid occur at the same $\mathrm{pH}$ value; therefore the anion dissociation constants calculated are the same.

As in previous work, allowance was made for acid neutralized by the small amount of combined cation in the purified fibers. The hydrogen-ion equivalence of their cation content, as shown by electrodialysis [9], was 0.015 millimole per gram, less than one-half as great as in the wool used in the earlier work. As previously explained, the validity of this procedure is in question only when small amounts of acids of high affinity are combined; since this occurs at very high dilution of the acid, it is possible that a fraction of the combined cations remains on the fiber in competition with the few hydrogen ions present. At most a very small number of the measurements in this paper were made under conditions admitting such a possibility; therefore the full correction has been applied to all the data.

When solutions more concentrated than $0.02 \mathrm{M}$ were used, the usual corrections were made for the selective absorption of water from the solution by the fibers, and where necessary, for the effects of any hydrolysis undergone by the wool. (See 4 in this section.)

\section{SOURCE OF ACIDS AND SPECIFIC PROCEDURES}

Except where particularized further below, commercial cp products were employed, and all procedures used were the same as those previously described $[12,15,16]$.

(1) Sulfamic and sulfosalicylic acids were Eastman products recrystallized from water to remove sulfuric acid present as an impurity.

(2) Naphthalene- $\beta$-sulfonic acid (Eastman) was separated from traces of naphthalene by filtration of the moderately dilute stock solutions.

(3) Isoamylsulfonic acid was prepared from the sodium salt (Eastman) by precipitation with lead nitrate and treatment of the lead salt, suspended in water, with hydrogen sulfide.

(4) Pyrophosphoric acid was prepared from tetrasodium pyrophosphate (Monsanto) by precipitation with lead nitrate and treatment of the lead salt, suspended in water, with hydrogen sulfide. Determination of the titration curve of the free acid showed that two equivalents of hydrogen ion (one-half of the available acidity) are almost completely dissociated in the $\mathrm{pH}$ range above 2.0 and that the third and fourth hydrogens are practically undissociated in solutions below $\mathrm{pH}$ 4.8. Since most of the measurements made with this acid fall between these two values, the curve obtained represents combination of wool with the divalent dihydrogen pyrophosphate ion. Assays of solutions of this acid were made by titrating to an electrometric endpoint at $\mathrm{pH} 4.8$ and calculating the results as two equivalents per mole. Tests showed that the material was not appreciably hydrolyzed at $0^{\circ} \mathrm{C}$ during the time required for the attainment of equilibrium with the wool.

(5) Dodecylsulfonic acid was prepared from dodecylsulfonyl chloride made by the method of Sprague and Johnson [10] from lauryl bromide (Eastman). The sulfonyl chloride was hydrolyzed by refluxing with $1.5 \mathrm{M}$ sodium hydroxide, and the lead salt was precipi- 
tated with lead acetate after acidifying the mixture with acetic acid. The free acid was obtained from the lead salt by the method of Noller and Gordon [6]. Special care was required to assure the removal of all perceptible traces of hydrochloric acid in this procedure. The affinity of dodecylsulfonate ion for wool is so great that the presence of even small amounts of hydrochloric acid will affect materially the $\mathrm{pH}$ at which a given amount of dodecylsulfonic acid is combined; - that is, the $\mathrm{pH}$ will be determined by the hydrochloric acid present rather than by the extremely small proportion of dodecylsulfonic acid left uncombined.

Concentrated solutions of this acid appear to be incompletely dissociated [18], but a good titration end point may be obtained with bromocresol purple or bromothymol blue.

(6) Dodecylsulfuric acid and octylsulfuric acid were prepared from sodium salts kindly supplied by the research laboratories of E. I. du Pont de Nemours \& Co., Inc. The first was precipitated with lead nitrate and the lead salt was decomposed with hydrogen sulfide; the second was precipitated as the barium salt which was subsequently decomposed with dilute sulfuric acid. It was necessary to coagulate the sulfide precipitate by adding ethanol, subsequently removed by vacuum distillation.

(7) Diphenylsulfonic acid (Monsanto) was separated from sulfuric acid, present as an impurity, by precipitating its silver salt and decomposing it with hydrogen sulfide. The silver sulfide was filtered off after coagulating it by the addition of alcohol, which was subsequently distilled off in vacuum at low temperature.

(8) $p$-Diphenylbenzenesulfonic acid was prepared from its sodium salt (Eastman) by treatment similar to that described for diphenylsulfonic acid.

(9) Isopropylnaphthalenesulfonic acid was prepared from its sodium salt (the commercial product Aerosol OS) by precipitation with lead nitrate, and treatment of the lead salt, suspended in 50 percent ethanol, with hydrogen sulfide followed by concentration in vacuum at low temperature. The starting material consisted of mixed isomers.

(10) Anthraquinone- $\beta$-sulfonic acid was prepared from its sodium salt (Eastman) by precipitating the lead salt and treating it, suspended in 50 percent ethanol, with a small excess of hydrochloric acid. Silver oxide was added in small portions until no further precipitation of silver chloride occurred. The excess silver at this point was determined by the Volhard method, and an equivalent quantity of hydrochloric acid was added to precipitate it. Most of the alcohol was then removed by vacuum distillation at room temperature. The free acid is not entirely stable in concentrated aqueous solutions, being transformed slowly to a substance which undergoes a color change (to red) in the $\mathrm{pH}$ range 6 to 7 . However, very little change occurred in the interval required by the measurements.

(11) $p$-hydroxyazobenzene- $p^{\prime}$-sulfonic acid was prepared from its sodium salt (Eastman) by precipitation with lead nitrate from a saturated solution. Further stages of the preparation were the same as for anthraquinone- $\beta$-sulfonic acid. This dye, unlike Orange II, which it somewhat resembles in structure, apparently forms no complexes with silver ion. Thus, use can be made of silver oxide in preparing the acid form. 
(12) Sodium metaphosphate was prepared by strongly heating sodium dihydrogen phosphate, as in the method of Briggs [2]. The sodium glass obtained by rapidly cooling the fused material dissolved slowly in cold water $\left(0^{\circ} \mathrm{C}\right)$ without hydrolyzing. Titration with hydrochloric acid at $23^{\circ} \mathrm{C}$ showed that the amount of hydrogen ion combined was only one-third as great as the sodium or phosphorus present. Thus, two-thirds of the hydrogen in hexametaphosphoric acid is strongly acidic, as Hisar has also found [5]. The apparent $\mathrm{pK}$ at $23^{\circ} \mathrm{C}$ of the weakly acidic group is 2.28 . The sodium glass is therefore regarded as trisodium trimetaphosphate, or a higher polymer of this compound, consistent with the findings of Partridge, Hicks, and Smith [7]. Although this interpretation differs from that of Briggs [2], it is not inconsistent with his data.

The experimental solutions were made up by adding various amounts of hydrochloric acid to solutions of a constant amount of the sodium glass. They thus differ from the other experimental solutions in containing, in addition to hydrogen ion and anion (monohydrogen trimetaphosphate or a polymer), equivalent amounts of sodium chloride plus residual amounts of the dissolved glass.

(13) The acid form of Orange II was prepared as previously described [16]. When stored in Pyrex bottles in the cold, no insoluble impurity was formed.

\section{MEASUREMENT OF REVERSIBILITY OF THE COMBINATION WITH ACIDS}

A number of the measurements were obtained by first allowing the wool to come into equilibrium with a solution which initially contained a relatively high concentration of acid, determining the amount combined by the usual methods, and then diluting a known volume of the solution (which still contained all the wool) with a known volume of water. After a suitable interval, a second aliquot of the solution was titrated, and the acid given off by the fiber was calculated. The acid remaining on the fiber was then entered in the table against a new $\mathrm{pH}$ determination. Measurements made in this way thus represent approach to a final state from the opposite direction to that represented by the other measurements.

\section{ALLOWANCE FOR THE EFFECTS OF HYDROLYTIC DECOMPOSITION}

When amide bonds in the wool are hydrolyzed, the apparent acidbinding capacity as measured by our method increases by an amount corresponding to the ammonia liberated. Accordingly, a correction for the effect of this ammonia was made wherever required. If peptide bonds also are hydrolyzed and soluble decomposition products only are formed, no additional correction is required except for the decrease in weight of the undissolved protein, which is usually negligible. However, if some insoluble decomposition products are formed, a real increase in acid-binding power may be observed. In earlier work with hydrochloric acid, only small amounts of ammonia (negligible at $0^{\circ} \mathrm{C}$ ) were formed, even with high concentrations of acid; of other dissolved products only traces could be detected. With acids of higher affinity, however, ammonia is liberated more rapidly; it may be detected in experiments made at $25^{\circ} \mathrm{C}$ or $50^{\circ} \mathrm{C}$ with initial con- 
centrations of certain acids as low as $0.005 M$. The necessary corrections (table 1) show a tendency to increase as the affinity of the anion of the acid increases, and have reached extreme values for the most concentrated solutions ( $0.05 M$ or less) of over 0.3 millimole per gram of wool in equilibration experiments requiring 3 to 4 days at $50^{\circ} \mathrm{C}$. Even at room temperature these acids have a noticeably destructive effect. Analyses for ammonia were made by the usual alkaline distillation, as well as by adsorption of the ammonia on

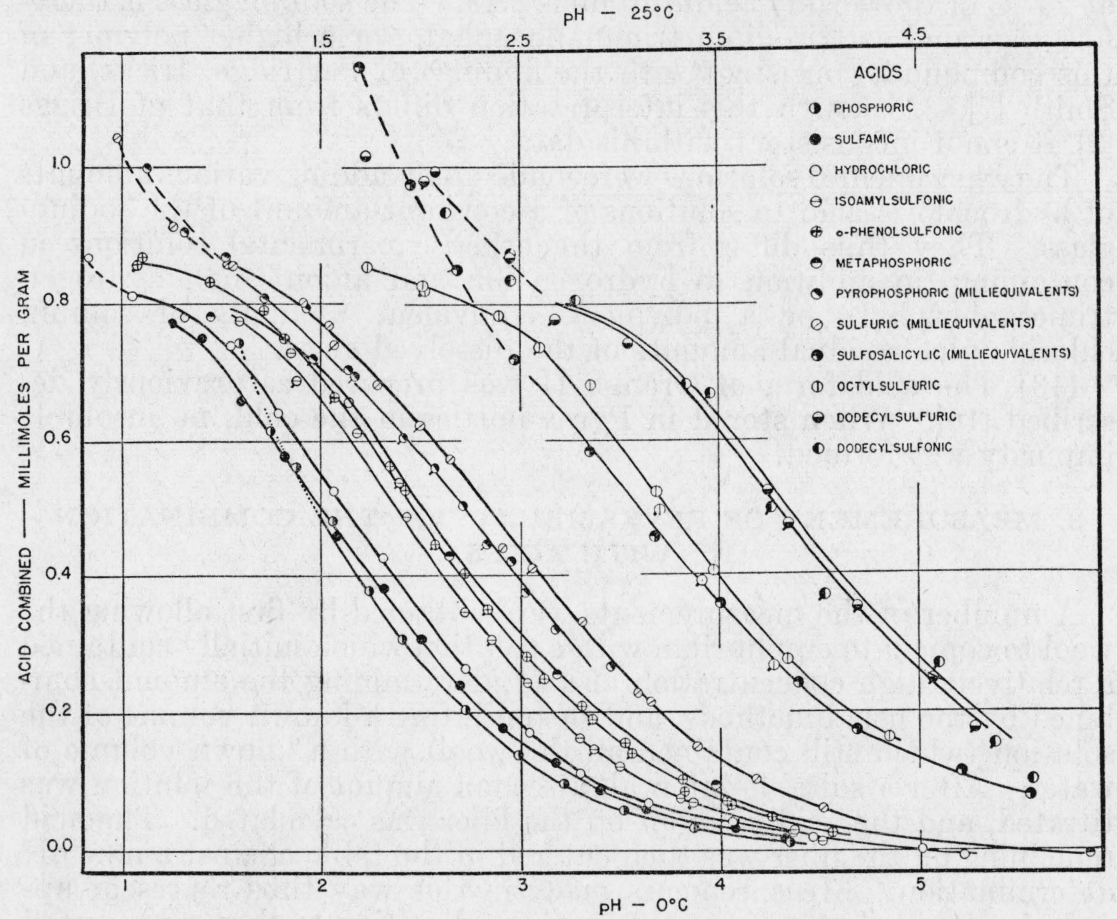

Figure 1.-Combination of wool protein with 12 different strong acids at $0^{\circ}$ or $25^{\circ} \mathrm{C}$.

The $\mathrm{pH}$ scale at the bottom of the figure applies to the data obtained at $0^{\circ} \mathrm{C}$. The scale at the top of the figure refers to the data obtained at $25^{\circ} \mathrm{C}$. The significance of the tagged circles is described in the text.

permutit, followed by alkaline distillation. The good agreement between the methods indicates that the ammonia found is a primary decomposition product rather than the product of secondary breakdown of dissolved protein during the alkaline distillation. Kjeldahl digestion of other aliquots has shown that appreciable amounts of nonammonia nitrogen are also present; thus, considerable hydrolysis of peptide bonds has probably occurred in these extreme cases. The corrections applied for the effects of hydrolysis at or near the tops of the curves obtained with the acids of highest affinity at $25^{\circ}$ and $50^{\circ} \mathrm{C}$ may therefore be inadequate to transform the data to values characteristic of the unchanged wool. It is in these cases, especially, that the flattening in the neighborhood of 0.83 to 0.84 millimole per gram combined, which characterizes the curves for the acids of lower affinity, disappears. 


\section{RESULTS AND DISCUSSION}

\section{TITRATION CURVES}

The data obtained with 18 different acids at 1 or more of 3 different temperatures are summarized in table 1 and are represented graphically in figures $1\left(0^{\circ}\right.$ and $\left.25^{\circ} \mathrm{C}\right)$ and $2\left(50^{\circ} \mathrm{C}\right)$. With most of the acids used the data represented have been obtained by approaching equilibrium from both sides, that is, by starting with fibers which are combined with more than their equilibrium amount of acid as well as with fibers that are initially uncombined. The "reversed" measurements are indicated in table 1 by italics, and by tagged circles in the figures. It is clear that the sets of data obtained in both ways are

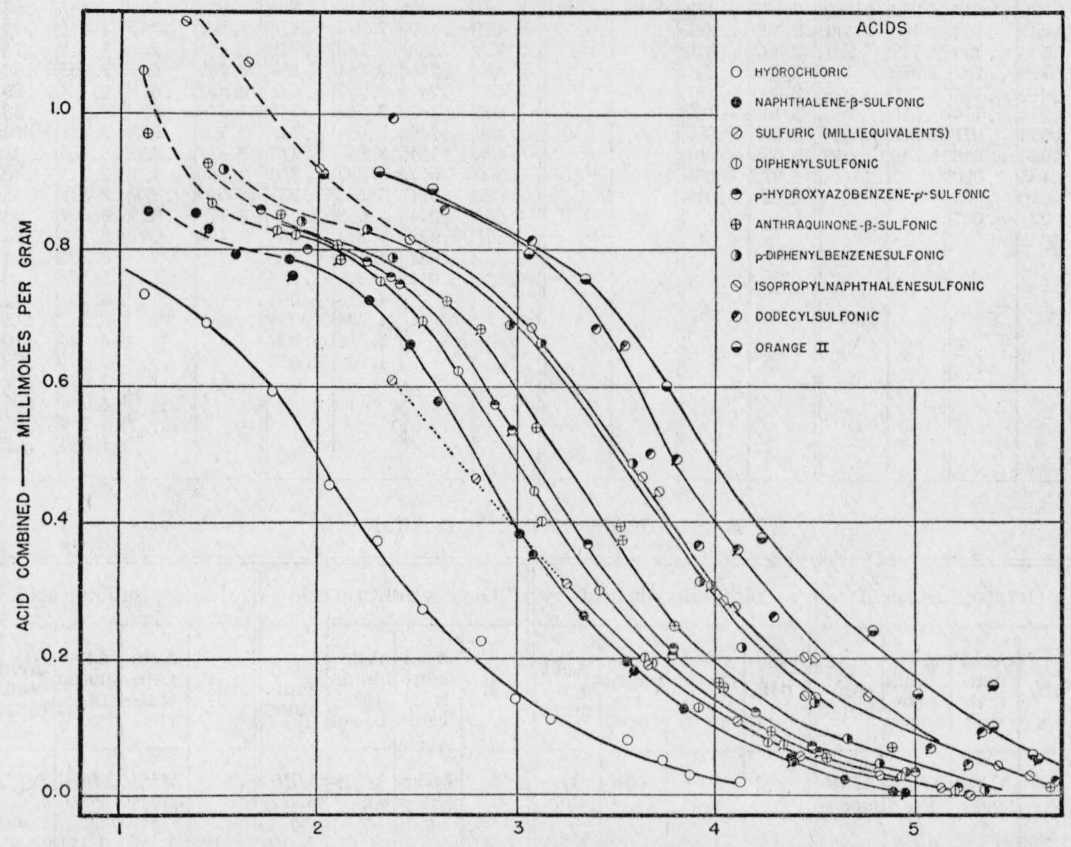

$\mathrm{PH}$

FIGURE 2.-Combination of wool protein with 10 different strong acids as a function of $\mathrm{pH}$ at $50^{\circ} \mathrm{C}$.

The significance of the tagged circles is described in the text.

consistent with one another. This agreement constitutes proof that the anion-wool associations measured are fully reversible, and that the wool in combining with acid suffers no permanent alteration until amounts which approach 0.8 millimole per gram are combined. ${ }^{3}$ In addition, such agreement eliminates the possibility that the results obtained are affected by the presence of acid impurities which differ appreciably in affinity from the substance under investigation. Other indications of the reversibility of the combination with acid have already been cited. Taken as a whole, they fully justify a theoretical treatment based on equilibrium considerations [12, 16].

${ }^{3}$ Data obtained with $\beta$-naphthoquinone-4-sulfonic acid did not fulfill this criterion of reversibility except for small amounts combined, and are therefore omitted from this paper. This exceptional behavior is consistent with the known reactivity of quinones with proteins. It is noteworthy that this dye changed color on combining with the fibers, and gave a curve of combination having an appreciably lower degree of dependence on $\mathrm{pH}$ than did all the other acids. 


\section{Journal of Research of the National Bureau of Standards}

TABLE 1.-Combination of wool"protein with various strong acids, in the absence of added salt

[Only new measurements, obtained with a single batch of wool, are included here. Italicized entries represent equilibria approached from the side of greater amounts combined]

A. RESULTS OBTAINED AT $0^{\circ} \mathrm{C}$

\begin{tabular}{|c|c|c|c|c|c|c|c|c|c|c|c|c|c|c|c|}
\hline \multicolumn{2}{|c|}{$\begin{array}{l}\text { Hydro- } \\
\text { chloric } \\
\text { acid }\end{array}$} & \multicolumn{2}{|c|}{$\begin{array}{l}\text { a Phos- } \\
\text { phoric } \\
\text { acid }\end{array}$} & \multicolumn{2}{|c|}{$\begin{array}{l}\text { Sulfamic } \\
\text { acid }\end{array}$} & \multicolumn{2}{|c|}{$\begin{array}{l}\text { b Meta- } \\
\text { phosphoric } \\
\text { acid }\end{array}$} & \multicolumn{2}{|c|}{$\begin{array}{l}\text { Isoamyl- } \\
\text { sulfonic } \\
\text { acid }\end{array}$} & \multicolumn{2}{|c|}{$\begin{array}{l}\text { - Sulfuric } \\
\text { acid }\end{array}$} & \multicolumn{2}{|c|}{$\begin{array}{l}\text { O Pyro- } \\
\text { phosphoric } \\
\text { acid }\end{array}$} & \multicolumn{2}{|c|}{$\begin{array}{l}\text { o-Phenol- } \\
\text { sulfonic } \\
\text { acid }\end{array}$} \\
\hline $\begin{array}{l}\mathrm{pH} \\
0.830 \\
1.051 \\
1.315 \\
1.546 \\
1.788 \\
2.061 \\
2.308 \\
2.716 \\
3.040 \\
3.294 \\
3.636 \\
4.060 \\
4.465 \\
5.010 \\
5.22\end{array}$ & \begin{tabular}{|c|} 
Milli- \\
moles $/$ \\
$g$ \\
0.871 \\
.814 \\
.782 \\
.740 \\
.643 \\
.528 \\
.430 \\
.267 \\
.174 \\
.14 \\
.071 \\
.043 \\
.020 \\
.003 \\
-.007
\end{tabular} & \begin{tabular}{|c}
$\mathrm{pH}$ \\
1.584 \\
1.745 \\
1.870 \\
2.053 \\
2.232 \\
2.400 \\
2.712 \\
2.980 \\
3.210 \\
3.510 \\
3.869 \\
4.349
\end{tabular} & 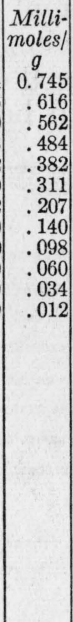 & $\begin{array}{l}\mathrm{pH} \\
1.253 \\
1.402 \\
1.598 \\
1.818 \\
2.077 \\
2.305 \\
2.493 \\
2.690 \\
2.905 \\
3.169 \\
3.395 \\
3.629 \\
4.07 \\
4.32\end{array}$ & $\begin{array}{c}\text { Milli- } \\
\text { moles/ } \\
g \\
0.773 \\
.751 \\
.660 \\
.579 \\
.472 \\
.385 \\
.310 \\
.244 \\
.181 \\
.129 \\
.089 \\
.061 \\
.034 \\
.018\end{array}$ & $\begin{array}{l}\mathrm{pH} \\
1.449 \\
2.639 \\
3.178 \\
3.976 \\
5.85\end{array}$ & $\begin{array}{c}\text { Milli- } \\
\text { moles/ } \\
g \\
0.783 \\
.433 \\
.259 \\
.097 \\
-.005\end{array}$ & $\begin{array}{l}\mathrm{pH} \\
1.592 \\
1.848 \\
1.872 \\
2.173 \\
2.388 \\
2.427 \\
2.713 \\
2.850 \\
3.030 \\
3.295 \\
3.366 \\
3.498 \\
3.800 \\
3.864 \\
4.066 \\
4.361 \\
4.367 \\
4.507\end{array}$ & \begin{tabular}{|c|} 
Milli- \\
moles \\
$g$ \\
0.776 \\
.730 \\
.690 \\
.612 \\
.540 \\
.490 \\
.350 \\
.310 \\
.247 \\
.166 \\
.149 \\
.124 \\
.077 \\
.071 \\
.044 \\
.028 \\
.031 \\
.014
\end{tabular} & $\begin{array}{l}\mathrm{pH} \\
0.97 \\
1.26 \\
1.53 \\
1.56 \\
1.81 \\
2.044 \\
2.066 \\
2.281 \\
2.492 \\
2.645 \\
2.901 \\
3.056 \\
3.303 \\
3.585 \\
3.653 \\
3.884 \\
4.180 \\
4.233 \\
4.513 \\
4.734 \\
4.791 \\
4.95\end{array}$ & $\begin{array}{c}M- \\
e q / g \\
1.045 \\
0.944 \\
.860 \\
.857 \\
.804 \\
.770 \\
.742 \\
.683 \\
.624 \\
.573 \\
.445 \\
.415 \\
.336 \\
.231 \\
.219 \\
.153 \\
.105 \\
.102 \\
.063 \\
.035 \\
.034 \\
.022\end{array}$ & $\begin{array}{c}\mathrm{pH} \\
1.126 \\
1.318 \\
1.525 \\
1.711 \\
1.924 \\
1.961 \\
2.135 \\
2.169 \\
2.359 \\
2.570 \\
2.708 \\
2.827 \\
2.950 \\
3.020 \\
3.170 \\
3.450\end{array}$ & $\begin{array}{l}M- \\
e q / g \\
1.006 \\
0.907 \\
.870 \\
.809 \\
.795 \\
.722 \\
.705 \\
.698 \\
.611 \\
.561 \\
.505 \\
.450 \\
.424 \\
.379 \\
.325 \\
.269\end{array}$ & $\begin{array}{l}\mathrm{pH} \\
0.708 \\
1.075 \\
1.106 \\
1.389 \\
1.445 \\
1.715 \\
1.812 \\
2.068 \\
2.134 \\
2.341 \\
2.418 \\
2.565 \\
2.714 \\
2.882 \\
3.027 \\
3.111 \\
3.302 \\
3.358 \\
3.521 \\
3.552 \\
3.806 \\
3.821 \\
4.030 \\
4.055 \\
4.256 \\
4.584\end{array}$ & $\begin{array}{c}\text { Milli- } \\
\text { moles/ } \\
g \\
0.893 \\
.860 \\
.869 \\
.887 \\
.833 \\
.792 \\
.750 \\
.666 \\
.657 \\
.563 \\
.530 \\
.450 \\
.403 \\
.355 \\
.285 \\
.253 \\
.213 \\
.193 \\
.159 \\
.143 \\
.106 \\
.097 \\
.074 \\
.068 \\
.051 \\
.027\end{array}$ \\
\hline
\end{tabular}

B. RESULTS OBTAINED AT $25^{\circ} \mathrm{C}$

\begin{tabular}{|c|c|c|c|c|c|c|c|c|c|c|c|c|c|c|c|}
\hline \multicolumn{4}{|c|}{ Octylsulfuric acid } & \multicolumn{4}{|c|}{ Sulfosalicylic acid } & \multicolumn{4}{|c|}{ Dodecylsulfuric acid } & \multicolumn{4}{|c|}{ Dodecylsulfonic acid } \\
\hline $\mathrm{pH}$ & $\begin{array}{l}\text { Acid } \\
\text { neu- } \\
\text { tral- } \\
\text { ized }\end{array}$ & $\begin{array}{c}\text { Am- } \\
\text { monia } \\
\text { pro- } \\
\text { duced }\end{array}$ & $\begin{array}{l}\text { Acid } \\
\text { com- } \\
\text { bined }\end{array}$ & $\mathrm{pH}$ & $\begin{array}{l}\text { Acid } \\
\text { neu- } \\
\text { tral- } \\
\text { ized }\end{array}$ & $\begin{array}{c}\text { Am- } \\
\text { monia } \\
\text { pro- } \\
\text { duced }\end{array}$ & $\begin{array}{l}\text { Acid } \\
\text { com- } \\
\text { bined }\end{array}$ & $\mathrm{pH}$ & $\begin{array}{l}\text { Acid } \\
\text { neu- } \\
\text { tral- } \\
\text { ized }\end{array}$ & $\begin{array}{c}\text { Am- } \\
\text { monia } \\
\text { pro- } \\
\text { duced }\end{array}$ & $\begin{array}{l}\text { Acid } \\
\text { com- } \\
\text { bined }\end{array}$ & $\mathrm{pH}$ & $\begin{array}{l}\text { Acid } \\
\text { neu- } \\
\text { tral- } \\
\text { ized }\end{array}$ & $\begin{array}{c}\text { Am- } \\
\text { monia } \\
\text { pro- } \\
\text { duced }\end{array}$ & $\begin{array}{l}\text { Acid } \\
\text { com- } \\
\text { bined }\end{array}$ \\
\hline $\begin{array}{l}1.735 \\
2.007 \\
2.019 \\
2.397 \\
2.595 \\
2.858 \\
3.180 \\
3.199 \\
3.477 \\
3.412 \\
3.84 \\
3.77 \\
4.35\end{array}$ & $\begin{array}{c}\text { Milli- } \\
\text { moles/ } \\
g \\
0.875 \\
.840 \\
\\
\end{array}$ & $\mid \begin{array}{c}\text { Milli- } \\
\text { moles } \\
g \\
0.020 \\
.011\end{array}$ & \begin{tabular}{|c|} 
Miuli- \\
moles $/$ \\
$g$ \\
0.855 \\
.829 \\
.821 \\
.784 \\
.757 \\
.681 \\
.525 \\
.499 \\
.412 \\
.896 \\
.281 \\
.272 \\
.167
\end{tabular} & $\begin{array}{l}1.082 \\
1.388 \\
1.696 \\
1.952 \\
2.188 \\
2.470 \\
2.850 \\
3.175 \\
3.504 \\
3.89 \\
4.19 \\
4.71 \\
5.06\end{array}$ & $\begin{array}{l}M- \\
e q / g \\
1.620 \\
1.407 \\
1.169 \\
0.986\end{array}$ & 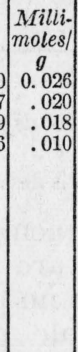 & $\begin{array}{c}M- \\
e q / g \\
1.594 \\
1.387 \\
1.151 \\
0.976 \\
.850 \\
.719 \\
.584 \\
.460 \\
.364 \\
.246 \\
.177 \\
.117 \\
.081\end{array}$ & $\begin{array}{l}1.727 \\
2.020 \\
2.078 \\
2.451 \\
2.678 \\
3.044 \\
3.743 \\
3.85 \\
4.19 \\
4.57 \\
4.78\end{array}$ & 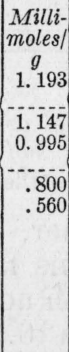 & $\mid \begin{array}{c}\text { Milli- } \\
\text { moles } \\
g \\
0.177 \\
.152 \\
.128 \\
.058 \\
.034\end{array}$ & \begin{tabular}{|c|} 
Milli- \\
moles \\
$g$ \\
1.016 \\
0.980 \\
.995 \\
.867 \\
.777 \\
.742 \\
.526 \\
.478 \\
.358 \\
.254 \\
.181 \\
\end{tabular} & $\begin{array}{l}2.129 \\
2.452 \\
2.459 \\
2.778 \\
3.338 \\
3.459 \\
3.760 \\
4.03 \\
4.60 \\
4.88 \\
5.08 \\
5.52 \\
5.67\end{array}$ & $\begin{array}{c}\text { Milli- } \\
\text { moles/ } \\
g \\
1.055 \\
00.881 \\
.698\end{array}$ & $\mid \begin{array}{c}\text { Milli- } \\
\text { moles } / \\
g \\
0.121 \\
.047 \\
.016\end{array}$ & $\mid \begin{array}{c}\text { Milli- } \\
\text { moles } \\
g \\
0.934 \\
.876 \\
.834 \\
.805 \\
.682 \\
.668 \\
.500 \\
.375 \\
.273 \\
.157 \\
.104 \\
.052 \\
.025\end{array}$ \\
\hline
\end{tabular}

a These measurements fall in the range of $\mathrm{pH}$ in which phosphoric acid is monobasic. b Salt was present in these experiments, as explained in the text.

- Calculations have been made on the basis of 2 equivalents per mole. 
TABLE 1.-Combination of wool protein with various strong acids, in the absence of added salt-Continued

\section{RESULTS OBTAINED AT $50^{\circ} \mathrm{C}$}

\begin{tabular}{|c|c|c|c|c|c|c|c|c|c|c|c|c|c|c|c|}
\hline \multicolumn{4}{|c|}{ Hydrochloric acid } & \multicolumn{4}{|c|}{$\begin{array}{c}\boldsymbol{\beta} \text {-Naphthalenesulfonic } \\
\text { acid }\end{array}$} & \multicolumn{4}{|c|}{ eSulfuric acid } & \multicolumn{4}{|c|}{ Diphenylsulfonic acid } \\
\hline $\mathrm{pH}$ & $\begin{array}{l}\text { Acid } \\
\text { neu- } \\
\text { tral- } \\
\text { ized }\end{array}$ & $\begin{array}{c}\text { Am- } \\
\text { monia } \\
\text { pro- } \\
\text { duced }\end{array}$ & $\begin{array}{l}\text { Acid } \\
\text { com- } \\
\text { bined }\end{array}$ & $\mathrm{pH}$ & $\begin{array}{l}\text { Acid } \\
\text { neu- } \\
\text { tral- } \\
\text { ized }\end{array}$ & $\begin{array}{c}\text { Am- } \\
\text { monia } \\
\text { pro- } \\
\text { duced }\end{array}$ & $\begin{array}{l}\text { Acid } \\
\text { com- } \\
\text { bined }\end{array}$ & $\mathrm{pH}$ & $\begin{array}{l}\text { Acid } \\
\text { neu- } \\
\text { tral- } \\
\text { ized }\end{array}$ & $\begin{array}{c}\text { Am- } \\
\text { monia } \\
\text { pro- } \\
\text { duced }\end{array}$ & $\begin{array}{c}\text { Acid } \\
\text { com- } \\
\text { bined }\end{array}$ & $\mathrm{pH}$ & $\begin{array}{l}\text { Acid } \\
\text { neu- } \\
\text { tral- } \\
\text { ized }\end{array}$ & $\begin{array}{c}\text { Am- } \\
\text { monia } \\
\text { pro- } \\
\text { duced }\end{array}$ & $\begin{array}{l}\text { Acid } \\
\text { com- } \\
\text { bined }\end{array}$ \\
\hline $\begin{array}{l}1.124 \\
1.435 \\
1.770 \\
2.055 \\
2.295 \\
2.525 \\
2.816 \\
2.990 \\
3.169 \\
3.553 \\
3.729 \\
3.870 \\
4.127\end{array}$ & $\begin{array}{c}\text { Milli- } \\
\text { moles/ } \\
g \\
0.800 \\
.730 \\
.609 \\
.471 \\
.384\end{array}$ & $\mid \begin{array}{c}\text { Milli- } \\
\text { moles/ } \\
g \\
0.064 \\
.036 \\
.016 \\
.014 \\
.010\end{array}$ & $\begin{array}{c}\text { Milli- } \\
\text { moles/ } \\
g \\
0.736 \\
.694 \\
.593 \\
.457 \\
.374 \\
.274 \\
.227 \\
.143 \\
.113 \\
.082 \\
.053 \\
.031 \\
.021\end{array}$ & $\begin{array}{l}1.147 \\
1.380 \\
1.447 \\
1.584 \\
1.864 \\
1.879 \\
2.254 \\
2.458 \\
2.597 \\
3.007 \\
3.078 \\
3.330 \\
3.554 \\
3.587 \\
3.842 \\
4.379 \\
4.395 \\
4.649 \\
4.890 \\
4.952\end{array}$ & $\begin{array}{c}\text { Milli- } \\
\text { moles/ } \\
g \\
1.032 \\
.956 \\
.833 \\
.805 \\
\\
\end{array}$ & $\begin{array}{c}\text { Milli- } \\
\text { moles/ } \\
g \\
0.175 \\
.081 \\
.039 \\
.016\end{array}$ & $\begin{array}{c}\text { Milli- } \\
\text { moles/ } \\
g \\
0.857 \\
.855 \\
.832 \\
.794 \\
.787 \\
.761 \\
.727 \\
.662 \\
.579 \\
.384 \\
.354 \\
.265 \\
.197 \\
.184 \\
.129 \\
.056 \\
.051 \\
.025 \\
.007 \\
.007\end{array}$ & $\begin{array}{l}2.370 \\
2.788 \\
3.244 \\
3.661 \\
4.107 \\
4.428 \\
4.823 \\
5.130 \\
5.28\end{array}$ & $\begin{array}{l}M- \\
e q / g \\
0.620\end{array}$ & $\mid \begin{array}{c}\text { Milli- } \\
\text { moles } / \\
g \\
0.010\end{array}$ & $\begin{array}{c}M- \\
e q / g \\
0.610 \\
.466 \\
.313 \\
.193 \\
.110 \\
.059 \\
.030 \\
.010 \\
.000\end{array}$ & $\begin{array}{l} \\
1.11 \\
1.46 \\
1.79 \\
1.88 \\
2.31 \\
2.52 \\
2.70 \\
3.08 \\
3.12 \\
3.41 \\
3.64 \\
3.68 \\
3.91 \\
4.25 \\
4.34 \\
4.93 \\
5.20\end{array}$ & $\begin{array}{c}\text { Milli- } \\
\text { moles/ } \\
g \\
1.398 \\
1.015 \\
0.875 \\
.772 \\
.630 \\
\end{array}$ & $\mid \begin{array}{c}\text { Milli- } \\
\text { moles/ } \\
g \\
0.334 \\
.146 \\
.042 \\
.018 \\
.007\end{array}$ & \begin{tabular}{|c} 
Milli- \\
moles/ \\
$g$ \\
1.064 \\
0.869 \\
.899 \\
.833 \\
.754 \\
.696 \\
.623 \\
.437 \\
.403 \\
.301 \\
.203 \\
.195 \\
.130 \\
.078 \\
.076 \\
.029 \\
.008
\end{tabular} \\
\hline
\end{tabular}

\begin{tabular}{|c|c|c|c|c|c|c|c|c|c|c|c|}
\hline \multicolumn{4}{|c|}{$\underset{p^{\prime} \text {-sulfonic acid }}{p \text {-Hydroxyazobenzene- }}$} & \multicolumn{4}{|c|}{$\begin{array}{l}\text { Anthraquinone- } \boldsymbol{\beta} \text { - } \\
\text { sulfonic acid }\end{array}$} & \multicolumn{4}{|c|}{$\begin{array}{l}\text { Isopropylnaphthalene- } \\
\text { sulfonic acid }\end{array}$} \\
\hline $\mathrm{pH}$ & $\begin{array}{l}\text { Acid } \\
\text { neu- } \\
\text { tral- } \\
\text { ized }\end{array}$ & $\begin{array}{c}\text { Am- } \\
\text { monia } \\
\text { pro- } \\
\text { duced }\end{array}$ & $\begin{array}{l}\text { Acid } \\
\text { com- } \\
\text { bined }\end{array}$ & $\mathrm{pH}$ & $\begin{array}{l}\text { Acid } \\
\text { neu- } \\
\text { tral- } \\
\text { ized }\end{array}$ & $\begin{array}{c}\text { Am- } \\
\text { monia } \\
\text { pro- } \\
\text { duced }\end{array}$ & $\begin{array}{l}\text { Acid } \\
\text { com- } \\
\text { bined }\end{array}$ & $\mathrm{pH}$ & $\begin{array}{l}\text { Acid } \\
\text { neu- } \\
\text { tral- } \\
\text { ized }\end{array}$ & $\begin{array}{c}\text { Am- } \\
\text { monia } \\
\text { pro- } \\
\text { duced }\end{array}$ & $\begin{array}{l}\text { Acid } \\
\text { com- } \\
\text { bined }\end{array}$ \\
\hline $\begin{array}{l}1.54 \\
1.71 \\
1.83 \\
1.94 \\
2.24 \\
2.36 \\
2.88 \\
2.97 \\
3.35 \\
3.78 \\
3.78 \\
4.20 \\
4.49 \\
4.53 \\
5.22 \\
5.27\end{array}$ & \begin{tabular}{c} 
Milli- \\
moles/ \\
$g$ \\
1.005 \\
0.924 \\
\hdashline .849 \\
.785 \\
\\
\end{tabular} & \begin{tabular}{c} 
Milli- \\
moles \\
$g$ \\
0.089 \\
.065 \\
\hdashline .048 \\
.025
\end{tabular} & \begin{tabular}{|c|} 
Milli- \\
moles/ \\
$g$ \\
0.916 \\
.859 \\
.905 \\
.801 \\
.783 \\
.760 \\
.573 \\
.537 \\
.369 \\
.207 \\
.217 \\
.124 \\
.070 \\
.065 \\
.013 \\
.003
\end{tabular} & $\begin{array}{l}1.138 \\
1.434 \\
1.807 \\
2.092 \\
2.106 \\
2.644 \\
2.811 \\
3.093 \\
3.512 \\
3.526 \\
3.792 \\
4.013 \\
4.635 \\
4.279 \\
4.557\end{array}$ & \begin{tabular}{c} 
Milli- \\
moles/ \\
$g$ \\
1.313 \\
1.142 \\
0.946 \\
.855 \\
\hdashline .742 \\
\hdashline .551
\end{tabular} & $\mid$\begin{tabular}{c} 
Milli- \\
moles/ \\
$g$ \\
0.342 \\
.215 \\
.095 \\
.048 \\
\hdashline .016 \\
.010
\end{tabular} & $\begin{array}{c}\text { Milli- } \\
\text { moles/ } \\
g \\
0.971 \\
.927 \\
.851 \\
.807 \\
.785 \\
.726 \\
.684 \\
.541 \\
.397 \\
.376 \\
.250 \\
.167 \\
.160 \\
.095 \\
.056\end{array}$ & $\begin{array}{l}1.307 \\
1.625 \\
1.994 \\
2.448 \\
3.065 \\
3.627 \\
3.710 \\
4.039 \\
4.096 \\
4.447 \\
4.447 \\
4.500 \\
4.500 \\
4.768 \\
5.42 \\
5.58\end{array}$ & \begin{tabular}{c} 
Milli- \\
moles/ \\
$g$ \\
1.525 \\
1.357 \\
1.026 \\
0.852 \\
.701 \\
.477 \\
\hdashline .295 \\
-.216
\end{tabular} & \begin{tabular}{c} 
Milli- \\
moles/ \\
$g$ \\
0.390 \\
.282 \\
.106 \\
.036 \\
.014 \\
.009 \\
\hdashline .009 \\
.011 \\
.009 \\
\end{tabular} & \begin{tabular}{|c} 
Milli- \\
moles/ \\
$g$ \\
1.135 \\
1.075 \\
.920 \\
.816 \\
.687 \\
.468 \\
.447 \\
.286 \\
.277 \\
.205 \\
.146 \\
.204 \\
.144 \\
.101 \\
.045 \\
.029
\end{tabular} \\
\hline
\end{tabular}


TABLE 1.-Combination of wool protein with various strong acids, in the absence of added salt-Continued

\begin{tabular}{|c|c|c|c|c|c|c|c|c|c|c|c|}
\hline \multicolumn{4}{|c|}{$\begin{array}{l}p \text {-Diphenylbenzene- } \\
\text { sulfonic acid }\end{array}$} & \multicolumn{4}{|c|}{ Dodecylsulfonic acid } & \multicolumn{4}{|c|}{ Orange II } \\
\hline $\mathrm{pH}$ & $\begin{array}{l}\text { Acid } \\
\text { neu- } \\
\text { tral- } \\
\text { ized }\end{array}$ & $\begin{array}{c}\text { Am- } \\
\text { monia } \\
\text { pro- } \\
\text { duced }\end{array}$ & $\begin{array}{l}\text { Acid } \\
\text { com- } \\
\text { bined }\end{array}$ & $\mathrm{pH}$ & $\begin{array}{l}\text { Acid } \\
\text { neu- } \\
\text { tral- } \\
\text { ized }\end{array}$ & $\begin{array}{c}\text { Am- } \\
\text { monia } \\
\text { pro- } \\
\text { duced }\end{array}$ & $\begin{array}{c}\text { Acid } \\
\text { com- } \\
\text { bined }\end{array}$ & $\mathrm{pH}$ & $\begin{array}{l}\text { Acid } \\
\text { neu- } \\
\text { tral- } \\
\text { ized }\end{array}$ & $\begin{array}{c}\text { Am- } \\
\text { monia } \\
\text { pro- } \\
\text { duced }\end{array}$ & $\begin{array}{c}\text { Acid } \\
\text { com- } \\
\text { bined }\end{array}$ \\
\hline $\begin{array}{l}1.525 \\
1.915 \\
2.239 \\
2.366 \\
2.956 \\
3.119 \\
3.577 \\
3.920 \\
3.972 \\
4.132 \\
4.499 \\
4.663 \\
4.821 \\
4.965 \\
5.02 \\
5.35\end{array}$ & \begin{tabular}{r} 
Milli- \\
moles/g \\
1.083 \\
0.914 \\
\hdashline .816 \\
.700 \\
\end{tabular} & \begin{tabular}{c} 
Milli- \\
moles/g \\
0.165 \\
.072 \\
\hdashline .027 \\
.010
\end{tabular} & $\begin{array}{r}\text { Milli- } \\
\text { moles } / g \\
0.918 \\
.842 \\
.820 \\
.789 \\
.690 \\
.665 \\
.489 \\
.315 \\
.310 \\
.219 \\
.137 \\
.084 \\
.048 \\
.031 \\
.016 \\
.009\end{array}$ & $\begin{array}{l}2.370 \\
2.629 \\
3.068 \\
3.397 \\
3.539 \\
3.666 \\
3.780 \\
3.916 \\
4.110 \\
4.290 \\
5.34 \\
5.08 \\
5.27 \\
5.72\end{array}$ & \begin{tabular}{r} 
Milli- \\
moles $/ g$ \\
1.224 \\
0.943 \\
\hdashline .701 \\
\end{tabular} & $\begin{array}{c}\text { Milli- } \\
\text { moles } / g \\
0.228 \\
.083 \\
.015\end{array}$ & $\begin{array}{c}\text { Milli- } \\
\text { moles/g } \\
0.996 \\
.861 \\
.815 \\
.686 \\
.662 \\
.503 \\
.495 \\
.368 \\
.362 \\
.263 \\
.095 \\
.070 \\
.047 \\
.022\end{array}$ & $\begin{array}{l}2.020 \\
2.300 \\
2.568 \\
3.053 \\
3.339 \\
3.749 \\
4.230 \\
4.790 \\
5.015 \\
5.395 \\
5.395 \\
5.595 \\
5.625 \\
5.835 \\
5.90 \\
6.16\end{array}$ & $\begin{array}{c}\text { Milli- } \\
\text { moles/g } \\
1.125 \\
1.072 \\
0.972 \\
.846 \\
.783 \\
.611 \\
\\
\end{array}$ & $\begin{array}{r}\text { Milli- } \\
\text { moles } / g \\
0.212 \\
.144 \\
.080 \\
.050 \\
.024 \\
.010\end{array}$ & $\begin{array}{c}\text { Milli- } \\
\text { moles/g } \\
0.913 \\
.928 \\
.892 \\
.796 \\
.759 \\
.601 \\
.379 \\
.244 \\
.151 \\
.163 \\
.102 \\
.061 \\
.055 \\
.029 \\
.007 \\
.006\end{array}$ \\
\hline
\end{tabular}

As in the results described earlier [16], considerable amounts in excess of 0.8 millimole appear to be bound when the most concentrated solutions of some of the acids are employed. Possible causes of this excess take-up of acid have been discussed in section II-4 and elsewhere [16]. The discussion of the affinity to wool of each of the anions dealt with in this paper is based entirely on the position with respect to the $\mathrm{pH}$ coordinate of the main S-shaped portion of the titration curve. In order to fix the position of this principal portion of each set of data, an effort has been made to draw continuous curves which represent the measurements fairly rather than to draw curves deliberately congruent with one another.

The curves which lie more than one to one and one-half $\mathrm{pH}$ units above the curve for hydrochloric acid must be considered as somewhat less accurately fixed by the experiments on three counts: (1) The pH values measured are those of very poorly buffered solutions; (2) the attainment of equilibrium is difficult, although the equilibrium curve can usually be fixed within narrow limits by comparing the measurements with the results of reversal experiments; (3) the relatively long periods at high temperatures required for attainment of equilibrium with acids of high affinity result in appreciable decomposition of the wool. This effect is intensified by the fact that decomposition is brought about more rapidly by acids of high affinity (table 1 ). Thus the choice of temperature at which each acid was investigated was governed in part by the desire to minimize as much as possible the aggregate effect of all of these factors as well as by the desire to obtain data at more than one temperature for a few acids of representatively different affinities. Acids having affinities equal to or greater than that of naphthalene- $\beta$-sulfonic acid were measured at either $25^{\circ}$ or $50^{\circ} \mathrm{C}$ rather than at $0^{\circ} \mathrm{C}$. 
The bearing of the results on the general question of the factors influencing affinity is considered in section IV of this paper, but matters of special interest concerning a few of the substances worked with are discussed individually below.

Hydrochloric acid no longer occupies an extreme position at the lowaffinity end of the diagram (fig. 1) as it did in the earlier paper. Two other acids give curves which indicate that their anions have affinities for wool lower than the affinity of chloride. Thus, titration curves of proteins, obtained with hydrochloric acid, are also clearly affected by the specific affinity of the chloride ion for the protein, and must not be regarded as representing a limiting case governed by the hydrogen-ion dissociation equilibrium of the carboxyl groups alone.

The lowest affinity is shown by phosphoric acid, and refers to the dihydrogen phosphate ion. The data for phosphoric acid are congruent with the other curves only for amounts combined of less than about 0.45 millimole per gram. This limitation is brought about by the fact that the first step in the dissociation of phosphoric acid is not complete in the range of $\mathrm{pH}$ below 2 . The presence of appreciable amounts of un-ionized acid results in the combination of additional amounts of acid in the undissociated form, as is found with weak acids generally [13].

The data at $0^{\circ} \mathrm{C}$ include measurements with two acids, sulfuric and pyrophosphoric, which are dibasic in the $\mathrm{pH}$ ranges of their respective curves. The amounts combined are therefore plotted as milliequivalents per gram instead of millimoles per gram. The curves for both indicate that their doubly charged anions have relatively high affinities, considering their molecular weights. A part, at least, of their high affinity may be due to the double charge, but this cannot constitute a full explanation. Thus, pyrophosphate has a lower affinity than sulfate although its molecular weight is considerably higher.

The curve obtained with metaphosphoric acid is not strictly comparable with those obtained with the other acids because of the presence of chloride, divalent monohydrogen trimetaphosphate, and trivalent metaphosphate ions. Its position is probably determined predominantly by the affinity of the divalent ion (or its dimer), since the concentration of this acid ion increases almost proportionally to the increase in hydrogen ion, as is the case when curves are obtained with strong acids in the absence of salt. The higher affinity of the basic trimetaphosphate ion is probably indicated by the titration curves obtained by Briggs for the soluble protein, serum albumen [2]. Because the titration curves for soluble proteins fall in a region of higher $\mathrm{pH}$ values [12], in these experiments the metaphosphate is nearly all in the fully ionized form. Thus the difference between the curves obtained by Briggs with hydrochloric acid and with hydrochloric acid plus metaphosphate is much larger than the difference found here with wool.

Among the data given for $25^{\circ} \mathrm{C}$ are measurements made with a third divalent anion, sulfosalicylate, commonly used as a protein precipitant. The affinity, as would be expected, is high but not so high as that of two other protein precipitants of higher molecular weight, picric and flavianic acids [16]. As in the case of flavianic acid, amounts far in excess of 0.8 milliequivalent per $\mathrm{g}$ are bound at low $\mathrm{pH}$ values. The anion present in this region of $\mathrm{pH}$ is the monovalent ion, and a $435456-42-6$ 
plateau near 0.8 millimole rather than at 0.8 milliequivalent would presumably be found.

The results shown for octylsulfuric, dodecylsulfonic, and dodecylsulfuric acids are the first given for strong aliphatic acids of high molecular weight. The first of these has a molecular weight slightly above that of naphthalenesulfonic acid; its affinity for wool is obviously higher. The affinities of the two larger molecules are among the highest so far measured. ${ }^{4}$ These results tend to contradict a suggestion made earlier [16], namely, that flat aromatic ions had higher affinities than linear ions, and are therefore discussed further elsewhere.

Among the data obtained at $50^{\circ} \mathrm{C}$ (fig. 2), the new measurements made with Orange II supersede those published earlier, since they have been corrected for the effect of the ash content of the fibers, and for the relatively rapid decomposition of the wool that occurs in the more concentrated solutions of this dye. The results therefore differ slightly from those published earlier.

With sulfuric acid the data at $50^{\circ} \mathrm{C}$ are much like those obtained at $0^{\circ} \mathrm{C}$ when the amounts combined are less than 0.3 milliequivalent per gram; beyond this point the increase in the amounts combined as the $\mathrm{pH}$ decreases is smaller than at the lower temperature. This anomaly may be attributed to the formation in the more acid solutions of the low-affinity bisulfate ion, a process which is enhanced by high temperature (the second dissociation constant of sulfuric acid decreases as the temperature is raised [4]). Thus at $\mathrm{pH}$ values below 3 there is considerably more bisulfate ion at $50^{\circ} \mathrm{C}$ than at $25^{\circ} \mathrm{C}$. This effect of high temperatures in effectually diminishing the affinity of sulfuric acid is of importance in dyeing, where not only high temperature but the presence of sulfate in addition to sulfuric acid favors the formation of bisulfate ion.

\section{THE SCALE OF ANION AFFINITIES}

In order to compare the results obtained with different acids on other than an empirical basis, it is necessary to reduce the positions of the curves to corresponding values of $\mathbf{K}_{\mathbf{A}}{ }^{\prime}$, the anion dissociation constant, defined in previous papers $[12,16]$. The resulting series of constants, calculated from the $\mathrm{pH}$ values at which half the maximum amounts are combined, not only furnishes an affinity scale to which a quantitative significance can be attached, but also makes possible the calculation of the effects of other environmental factors, such as the presence of salts, in terms of a mass-action effect, or of the effect of temperature, in terms of heats of dissociation. A slight modification [11] in the original method of calculation has recently been introduced in order to extend the range of usefulness of the previously formulated"equations. With this modification, the equations describe the relevant parts of the curves in full detail, and not merely the positions of the midpoints of the curves.

Using the new equations, values of $K_{\mathrm{A}}{ }^{\prime}$ for all the acids so far investigated have been calculated by employing the appropriate values of $K_{\mathrm{H}}{ }^{\prime}$ for each temperature [16]. The results of the calcula-

\footnotetext{
4 The high affinity of dodecylsulfate is of special interest in view of its recent use as a protein denaturant [1]. As Smith [8] has shown, the effect of this substance, unlike that of other protein denaturants, is to disrupt at least one protein into fragments. The hydrolytic effect of dodecylsulfuric acid on wool protein shown in table 1, and further described elsewhere [17], is the greatest of any acid so far investigated. Since, in general, the hydrolytic effect increases with the affinity, the drastic effects of dodecylsulfate on soluble proteins may be shared in greater or lesser degree by other compounds of high molecular weight.
} 
tion are assembled in table 2 . The newly calculated values of $-\log$ $K_{\mathrm{A}}{ }^{\prime}$ for acids measured previously are compared in the table with the values given earlier. The revised values are not far different from those given by the old equations, especially in the range of lower affinities. The larger differences are found with the anions of highest affinity, since there is a slightly smaller degree of dependence of $K_{A}{ }^{\prime}$ on the $\mathrm{pH}$ of half-maximum combination in the new equations.

\section{TABLE 2.-Calculation of anion affinity constants $\left(1 / K_{\mathrm{A}}^{\prime}\right)$ at three different temperatures}

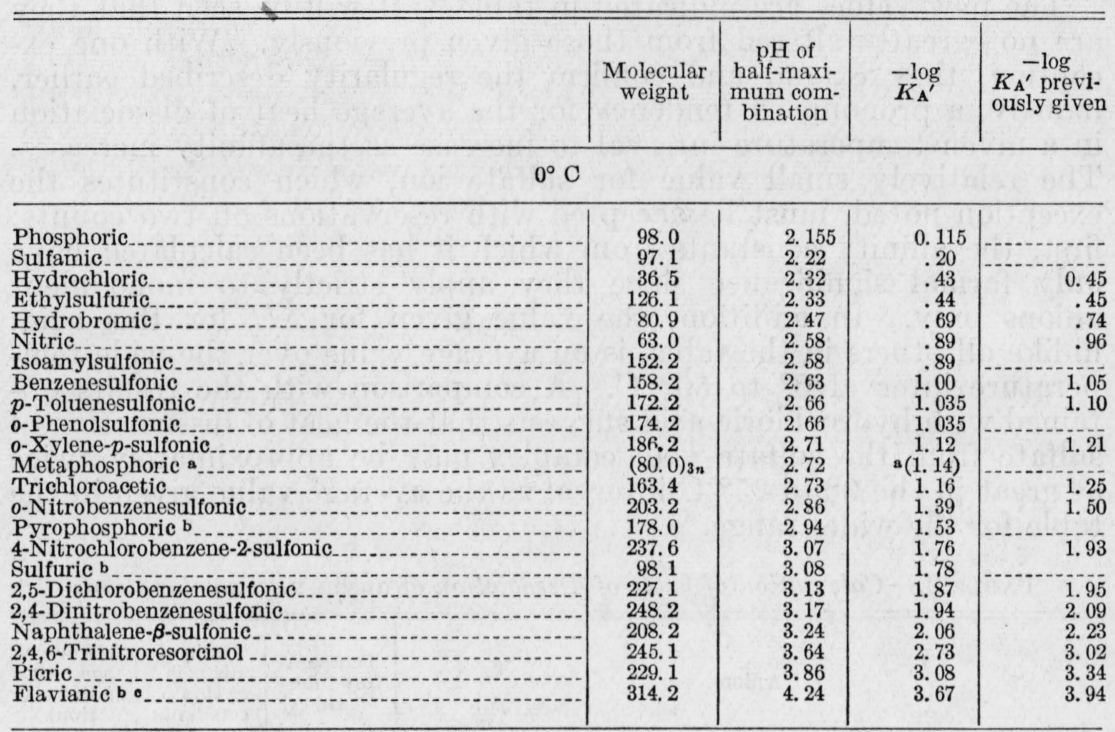

$25^{\circ} \mathrm{C}$

\begin{tabular}{|c|c|c|c|c|}
\hline Hydrochloric. & 36.5 & 2.16 & 0.265 & 0.31 \\
\hline Naphthalene- $\boldsymbol{\beta}$-sulfonic & 208. 2 & 3. 03 & 1.82 & 1. 97 \\
\hline Octylsulfuric & 210. 3 & 3.47 & 2. 56 & \\
\hline Sulfosalicylic.-1, & 218.1 & 3.34 & 2.34 & \\
\hline Picric & 229.1 & 3. 52 & 2. 64 & 2.89 \\
\hline Dodecylsulfuric & 266.4 & 4. 02 & 3.42 & \\
\hline Flavianic b & 314. 2 & 4.07 & 3.495 & 3. 77 \\
\hline Dodecylsulfonic c & 250.4 & $\begin{array}{r}4.08 \\
0.43\end{array}$ & 3.51 & \\
\hline Orange $110^{\circ}-x_{1}$ & 020.0 & 0.63 & 04.28 & 6.53 \\
\hline
\end{tabular}

$50^{\circ} \mathrm{C}$

Hydrochloric

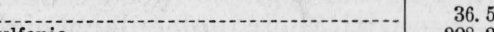

Sulfuric b.

Diphenylsulfonic

$p$-Hydroxyazobenzene- $p^{\prime}$-sulfonic

Anthraquinone- $\beta$-sulfonic

$p$-Diphenylbenzenesulfonic

iso-Propylnaphthalenesulfonic

Orange II.
Dodecylsulfonic

\begin{tabular}{|c|c|c|c|}
\hline 36.5 & 2.17 & 0.28 & 0.38 \\
\hline 208.2 & 2.95 & 1.68 & \\
\hline 98.1 & $\mathrm{~d} 2.97$ & 1.71 & \\
\hline 234.3 & 3.16 & 2.04 & \\
\hline 278.3 & 3. 24 & 2.18 & -... \\
\hline 288.3 & 3. 40 & 2.44 & -... \\
\hline 310.4 & 3. 70 & 2.93 & \\
\hline 250.3 & 3.74 & 2. 99 & - \\
\hline $\begin{array}{l}250.4 \\
328.3\end{array}$ & $\begin{array}{l}3.96 \\
4.17\end{array}$ & $\begin{array}{r}3.33 \\
+3.64\end{array}$ & 3.94 \\
\hline 328.3 & 4.16 & 0.04 & 3.94 \\
\hline
\end{tabular}

a The affinity indicated is minimal, for reasons explained in the text.

b The data given refer to combination with the divalent anion, but $K_{\mathrm{A}}$ ' is expressed, for purposes of comparison, as if the anion were monovalent.

- The affinity given is minimal because equilibrium may not have been attained

d Extrapolated from the lower (normal) portion of the titration curve. The actual midpoint $\mathrm{pH}$ was 2.92.

- The midpoint $\mathrm{pH}$ of the curve obtained with Orange II at $25^{\circ} \mathrm{C}$ has been reevaluated by applying a correction for ash to these data. Both values of $-\log K_{\mathrm{A}}$ are based on the reevaluated midpoint.

${ }^{1}$ This value is based on the revised midpoint $\mathrm{pH}$ given in the present paper. 


\section{Journal of Research of the National Bureau of Standard's}

\section{EFFECT OF TEMPERATURE ON AFFINITY}

The revised values of $K_{\mathrm{A}}{ }^{\prime}$ in table 2 entail a slight revision of the values previously given for $\Delta H$, the heat of dissociation of the anions from the anion-wool complex.

The assumptions which enter into calculations, from titration data at two temperatures, of $\Delta H$ for the dissociation of a hydrogen ion from the protein have been discussed elsewhere [15, especially p. 535 and footnote 11]. The same assumptions are now applied to the calculation of the heat of dissociation of anions as well.

The new values are indicated in table 3 ; it will be seen that they are not greatly altered from those given previously. With one exception, they extend and confirm the regularity described earlier, namely, a pronounced tendency for the average heat of dissociation in a given temperature interval to increase as the affinity increases. The relatively small value for sulfate ion, which constitutes the exception noted, must be accepted with reservations on two counts: first, the affinity constants from which it has been calculated have only formal significance since they apply strictly to monovalent anions only. In addition, the value given for $\Delta H$ for this acid, unlike all others in the table, is an average value over the wide temperature interval $0^{\circ}$ to $50^{\circ} \mathrm{C}$. A comparison with the results obtained with hydrochloric acid suggests that the heat of dissociation of sulfate from the sulfate-wool complex may be approximately twice as great in the $0^{\circ}$ to $25^{\circ} \mathrm{C}$ interval as the average value given in the table for the wider range.

TABLE 3.-Calculation of heats of dissociation of anion-wool combinations

\begin{tabular}{|c|c|c|c|}
\hline Anion & $\begin{array}{l}\text { Tempera- } \\
\text { ture inter- } \\
\text { val }\end{array}$ & $\begin{array}{l}\text { Ratio of } \\
\text { dissocia- } \\
\text { tion } \\
\text { constants }\end{array}$ & $\begin{array}{l}\Delta H \text { (aver- } \\
\text { age heat } \\
\text { of dissocia- } \\
\text { tion) }\end{array}$ \\
\hline Chloride.. & \multirow{3}{*}{$\begin{array}{l}{ }^{\circ} \mathrm{C} \\
0 \text { to } 25 \\
25 \text { to } 50 \\
0 \text { to } 50 \\
0 \text { to } 25 \\
25 \text { to } 50 \\
0 \text { to } 25 \\
25 \text { to } 50 \\
25 \text { to } 50\end{array}$} & \multirow{3}{*}{$\begin{array}{l}1.481 \\
0.975 \\
1.163 \\
1.755 \\
1.338 \\
2.75 \\
1.51 \\
>4.36\end{array}$} & \multirow{3}{*}{$\begin{array}{r}\text { Calories } \\
2,550 \\
-194 \\
532 \\
3,640 \\
2,220 \\
6,550 \\
3,180 \\
>11,300\end{array}$} \\
\hline $\begin{array}{l}\text { Sulfate } \\
\text { Naphthalene- } \boldsymbol{\beta} \text {-sulfonate }\end{array}$ & & & \\
\hline $\begin{array}{l}\text { Picrate } \\
\text { Dodecylsulfonate } \\
\text { Orange II }\end{array}$ & & & \\
\hline
\end{tabular}

\section{RELATION OF CHEMICAL STRUCTURE TO AFFINITY}

The data collected in table 2 reveal a pronounced tendency for the affinity of anions for wool to rise with their molecular weights. Thus, the question of the existence of any additional factors modifying this tendency may best be examined by comparing the affinities of different substances or classes of substances on the basis of their sizes or molecular weights rather than by comparing the affinities themselves. Such a comparison is facilitated by figure 3, in which quantities proportional to the free energy of binding of the anions $\left(-\log K_{\mathrm{A}}^{\prime}\right)$ of a large proportion of all the acids studied have been plotted against their respective molecular weights. Among the acids investigated, only the strong monobasic organic acids are represented in order to bring out simple relationships more clearly. To include as wide a range of molecular weights and of affinities as possible, data for two temperatures, $0^{\circ}$ and $50^{\circ} \mathrm{C}$, have been combined; a division between 
the data at the two temperatures has been effected arbitrarily, with only slight overlapping, at $\log K_{\mathrm{A}}^{\prime}$ approximately equal to -2 . Naturally it cannot be expected that there will be any continuity between the data obtained at the two different temperatures, but their combination serves to point a trend, and the data for each temperature may be compared among themselves.

The simple relations shown indicate an absence of any large specific effects of different substituents on the affinity within the two groups of acids represented. Thus, to point to only a few examples, $o$-phenolsulfonic and $p$-toluenesulfonic acids have nearly equal molecular weights and show practically identical affinities, although the properties of the $\mathrm{OH}$ and $\mathrm{CH}_{3}$ groups (such as polarity, polarizability, and resonance energy) differ markedly. Likewise the differences in the

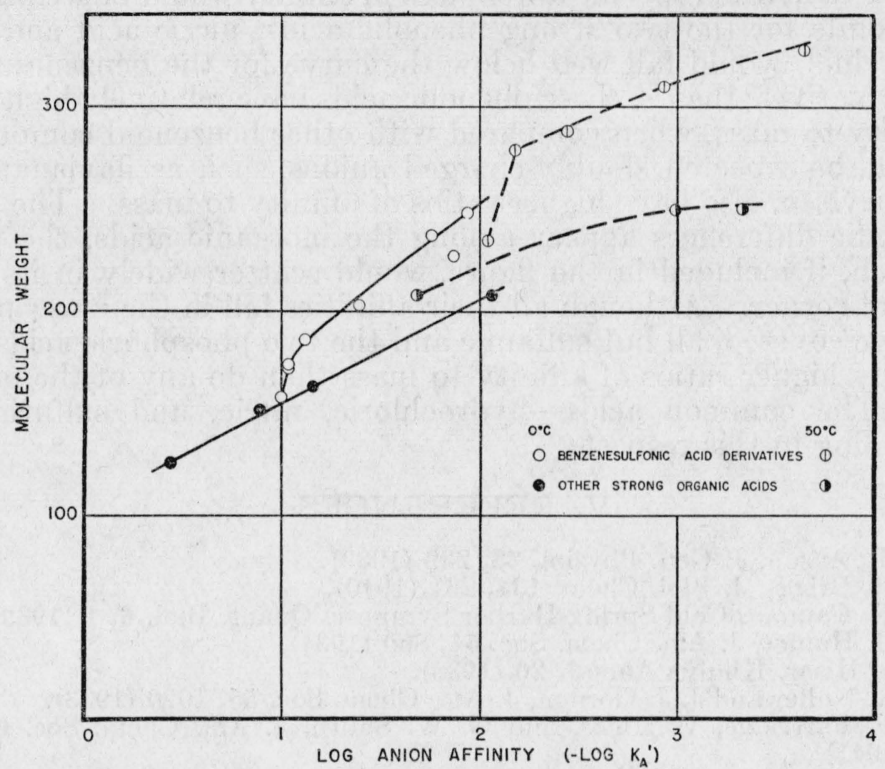

Figure 3.-Molecular weights and values of log affinity for strong monobasic organic acids.

Data for two temperatures, $0^{\circ}$ and $50^{\circ} \mathrm{C}$, are represented.

positions on the diagram of xylene-, nitrobenzene-, nitrochlorobenzene-, and dinitrobenzene-sulfonic acids are consistent with their relative molecular weights, despite similar large differences in the properties of their respective substituents. Such regularities in the relation between affinity and molecular weight as are shown in figure 3 imply that the effects of temperature on anion affinity are also fairly regular. This has already been indicated by the data presented in the preceding section.

However, the fact that the points in the diagram fall roughly into two groups is an indication that there are limits to the range of chemical structure within which marked specific effects fail to appear. Thus, among the strong organic acids, those which are benzenoid in character have lower affinities for wool than those which fall in other categories (aliphatic, naphthenoid, etc.). Since the simplest member of the benzenoid series, benzenesulfonic acid, falls on both curves, it 
cannot be maintained that the benzene ring itself has a particularly low ratio of affinity to mass. Rather, it appears that the mass added by a substituent to the benzene ring is relatively less effective in increasing the affinity to wool than is the same mass added to aliphatic or conjugated ring compounds. This reduced effectiveness appears even when the substituent added is another benzene ring, as is shown in the strictly homologous series benzenesulfonic, diphenylsulfonic, diphenylbenzenesulfonic acids. It does not appear, or appears less strongly, when the rings are conjugated, as in naphthalenesulfonic acid. The existence of similar effects is suggested by the scantier data obtained at $25^{\circ} \mathrm{C}$, which are not represented in the figure. The reason for these differences remains obscure.

If the affinities of other types of acids were included in figure 3 , the existence of marked specific differences in affinity would be well shown. Thus points for the two strong phenolic acids, picric acid and trinitroresorcinol, would fall well below the curve for the benzenesulfonic acid derivatives; that is, these phenolic acids have relatively high ratios of affinity to mass, when compared with other benzenoid compounds. As would be expected, doubly charged anions, such as flavianate and sulfosalicylate, also have higher ratios of affinity to mass. The greatest specific differences appear among the inorganic acids, the points for which, if included in the figure, would scatter widely in its lower left-hand corner. Although all their affinities fall in the lower part of the range covered, all but sulfamic and the two phosphoric acids have relatively higher ratios of affinity to mass than do any of the organic acids. The common acids-hydrochloric, nitric, and sulfuric-are outstanding in this respect.

\section{REFERENCES}

[1] M. L. Anson, J. Gen. Physiol. 23, 239 (1939).

[2] D. R. Briggs, J. Biol. Chem. 134, 261 (1940).

[3] R. K. Cannan, Cold Spring Harbor Symposia Quant. Biol. 6, 1 (1939).

[4] W. J. Hamer, J. Am. Chem. Soc. 54, 860 (1934).

[5] R. S. Hisar, Kimiya Ann. 3, 20 (1938).

[6] C. R. Noller and J. J. Gordon, J. Am. Chem. Soc. 55, 1090 (1933).

[7] E. P. Partridge, V. Hicks, and G. W. Smith, J. Am. Chem. Soc. 63, 454 (1941).

[8] E. Smith, J. Gen. Physiol. 24, 583 (1941); 24, 753 (1941).

[9] A. Sookne, C. H. Fugitt, and J. Steinhardt, J. Research NBS 25, 61 (1940) RP1314; Textile Research 10, 380 (1940); Am. Dyestuff Reptr. 29, 1333 (1940).

[10] J. M. Sprague and T. B. Johnson, J. Am. Chem. Soc. 59, 1837 (1937).

[11] J. Steinhardt, J. Research NBS 28, 191 (1942) RP1452.

[12] J. Steinhardt and M. Harris. J. Research NBS 24, 335 (1940) RP1286; Textile Research 10, 181 (1940); Am. Dyestuff Reptr. 29, 103 (1940).

[13] J. Steinhardt and M. Harris, Proc. Am. Soc. Biological Chemists XXXIV, J. Biol. Chem. 134, xcvii (1940).

[14] J. Steinhardt and M. Harris, Proc. Am. Soc. Biological Chemists XXXV, J. Biol. Chem. 140, cxxiv (1941). A more complete account is in preparation.

[15] J. Steinhardt, C. H. Fugitt, and M. Harris, J. Research NBS 25, 519 (1940) RP1343; Textile Research 11, 72 (1940); Am. Dyestuff Reptr. 29, 607 (1940).

[16] J. Steinhardt, C. H. Fugitt and M. Harris, J. Research NBS 26, 293 (1941) RP1377; Textile Research 11, 259-284 (1941); Am. Dyestuff Reptr. 30, 223 (1941).

[17] J. Steinhardt, J. Biol. Chem. 141, 995 (1941).

[18] P. van Rysselberghe, J. Phys. Chem. 43, 1049 (1939).

Washington, November 7, 1941. 